\title{
Fatores associados ao comportamento suicida em hospital psiquiátrico: estudo transversal
}

\author{
Factors associated with suicide behavior in psychiatric hospital: cross study
}

Factores asociados con el comportamiento del suicidio en el hospital psiquiátrico: estudio transversal

Krieger Rhelyni de Sousa Olinda ${ }^{1 *}$, Rosimeire Ferreira dos Santos ${ }^{1}$, Thiago Henrique Costa Marques $^{3}$.

\section{RESUMO}

Objetivo: Analisar os fatores associados ao comportamento suicida em Hospital Psiquiátrico. Métodos: Estudo transversal analítico realizado em hospital de referência para tratamento de transtornos mentais graves de Teresina, no período de fevereiro a maio de 2018. A amostra foi constituída por 142 pacientes com idade igual ou superior à 18 anos e que deram entrada no serviço de urgência após de tentativa de suicídio. Para coleta de dados foi utilizado o formulário de caracterização sociodemográfica, clínica e terapêutica. A análise foi realizada com base nos princípios da estatística descritiva e inferencial e o estudo atendeu aos preceitos éticos e legais, tendo parecer emitido pelo Comitê de Ética em Pesquisa da Universidade Federal do Piauí. Resultados: Prevaleceram pacientes do sexo masculino, com idade média de 34,4, de baixa escolaridade e procedentes de Teresina. Os fatores associados foram transtorno psiquiátrico, consumo de substâncias psicoativas e tentativas prévias. Associações significativas foram verificadas entre 0 comportamento e variáveis sociodemográficas, clínicas e terapêuticas como sexo, idade, tentativas prévias e internação psiquiátrica. Conclusão: Fluxos assistenciais devem ser prerrogativas frequentes nos serviços de saúde, visando a identificação precoce dos determinantes e implementação de estratégias voltadas para preservação da vida, redução de riscos e dos indicadores de mortalidade.

Palavras-chave: Prevenção e controle, Saúde mental, Suicídio.

\begin{abstract}
Objective: To analyze factors associated with suicidal behavior in a psychiatric hospital. Methods: Analytical cross-sectional study conducted at a referral hospital for the treatment of severe mental disorders in Teresina, from February to May 2018. The sample consisted of 142 patients aged 18 years or older and admitted to the urgency after attempted suicide. For data collection, the sociodemographic, clinical and therapeutic characterization form was used. The analysis was performed based on the principles of descriptive and inferential statistics and the study complied with the ethical and legal precepts, having an opinion issued by the Research Ethics Committee of the Federal University of Piauí. Results: Male patients with a mean age of 34.4, low education and from Teresina prevailed. The associated factors were psychiatric disorder, psychoactive substance use and previous attempts. Significant associations were found between behavior and sociodemographic, clinical and therapeutic variables such as gender, age, previous attempts and psychiatric hospitalization. Conclusion: Care flows should be frequent prerogatives in health services, aiming at early identification of determinants and implementation of strategies aimed at preserving life, risk reduction and mortality indicators.
\end{abstract}

Key words: Prevention and control, Mental health, Suicide.

${ }^{1}$ Universidade Federal do Piauí (UFPI), Teresina-Piauí. *E-mail: krieger_olinda@hotmail.com.

2 Instituto Federal do Piauí (IFPI), Teresina-Piauí. 


\section{RESUMEN}

Objetivo: analizar los factores asociados con el comportamiento suicida en un hospital psiquiátrico. Métodos: estudio transversal analítico realizado en un hospital de referencia para el tratamiento de trastornos mentales graves en Teresina, de febrero a mayo de 2018. La muestra consistió en 142 pacientes mayores de 18 años que ingresaron en el hospital. urgencia después de un intento de suicidio. Para la recolección de datos, se utilizó la forma de caracterización sociodemográfica, clínica y terapéutica. El análisis se realizó sobre la base de los principios de estadística descriptiva e inferencial y el estudio cumplió con los preceptos éticos y legales, con una opinión emitida por el Comité de Ética en Investigación de la Universidad Federal de Piauí. Resultados: prevalecieron los pacientes varones con una edad media de 34.4 años, baja educación y de Teresina. Los factores asociados fueron trastorno psiquiátrico, uso de sustancias psicoactivas e intentos previos. Se encontraron asociaciones significativas entre el comportamiento y las variables sociodemográficas, clínicas y terapéuticas como género, edad, intentos previos y hospitalización psiquiátrica. Conclusión: Los flujos de atención deben ser prerrogativas frecuentes en los servicios de salud, con el objetivo de identificar tempranamente los determinantes e implementar estrategias destinadas a preservar los indicadores de vida, reducción de riesgos y mortalidad.

Palabras clave: Prevención y control, Salud mental, Suicidio.

\section{INTRODUÇÃO}

O suicídio constitui um problema de saúde pública evitável e de abrangência mundial, tendo maior impacto em países de baixa e média renda por apresentar indicadores elevados de incidência e mortalidade, assim como por configurar a décima quinta principal causa de morte na população e a segunda mais frequente entre adolescentes e adultos jovens de ambos os sexos (SOUSA VS, et al., 2011; WORLD HEALTH ORGANIZATION, 2016).

Considerando um fenômeno complexo e multidimensional, o suicídio consiste em uma ação voluntária de autoextermínio, geralmente associado à um transtorno mental, sendo definido como ato deliberado, executado pelo próprio indivíduo, cuja intenção seja a morte de forma consciente e intencional, usando um meio que acredita ser efetivo (CALIXTO M, ZERBINI T, 2016).

De acordo com a etimologia, o suicídio deriva das palavras sui, de si mesmo, e caedes, ação de matar, representando, assim, matar a si mesmo. Estudos demonstram que a magnitude do problema pode ser mensurada pelos prejuízos econômicos, sociais e psicológicos para os familiares e para a sociedade, além do ônus econômico gerado para os serviços de saúde (WORLD HEALTH ORGANIZATION, 2014).

Embora evidenciado com maior frequência em subgrupos populacionais, os elevados indicadores relacionados ao comportamento refletem as deficiências das políticas públicas de saúde mental e a falta de fluxos com diretrizes e recomendações para o direcionamento da assistência, assim como para avaliação das situações de risco, tratamento adequado e suporte social (YARBOROUGH BHJ, et al., 2019).

Para a Organização Mundial da Saúde (OMS), anualmente mais de 800 mil pessoas cometem suicídio por ano, o que corresponde a uma taxa global ajustada de 10,7 casos por 100 mil habitantes. O Brasil segue os parâmetros mundiais, em que na maioria das vezes o comportamento suicida não é reconhecido como emergência, sendo agravado pelas limitações assistenciais e pela subnotificação que contribui para efetivação da prática e o aumento dos indicadores (WORLD HEALTH ORGANIZATION, 2016).

Assim, inúmeros estudos que foram desenvolvidos para estimar os coeficientes associados ao suicídio no Brasil, mostraram que entre os anos de 2000 a 2012, foram registrados 112.103 óbitos e que houve o aumento de $26,5 \%$ na taxa de mortalidade (MACHADO DB, SANTOS DN, 2015).

Ainda, estima-se que ocorrem em média 24 mortes por dia, havendo indícios de que essa estatística possa ser maior, uma vez que tentativas suicidas foram apresentadas como uma das principais causas para institucionalização no período de 1998 a 2014, com o total de 153.061 internações (BAHIA CA, et al., 2017). 
A literatura demonstra que comportamento suicida envolve diferentes condições como a ideação, o planejamento, a tentativa e o suicídio consumado, podendo ser diretamente influenciado por aspectos individuais, coletivos, sociais, psicológicos, culturais, biológicos e ambientais que interagem entre si e determinam o risco para o agravo (CARDOSO HF, et al., 2014; CALIXTO M, ZERBINI T, 2016; FÉLIX JC, et al., 2016).

Assim, a compreensão do comportamento suicida tornou-se multidisciplinar e a sua avaliação precoce é amplamente referenciada, visando direcionar políticas públicas efetivas, integradas, sustentáveis e baseadas em evidências, assim como nortear a prática assistencial e a definição de condutas preventivas e terapêuticas. De acordo com Muller AS, Pereira G e Zanon RB (2017), a prevenção envolve uma série de atividades que vão desde proporcionar as melhores condições de vida possíveis na infância e adolescência, passando pelo tratamento eficaz dos transtornos psiquiátricos, medidas de vigilância, controle ambiental e identificação dos fatores de risco.

Frente ao exposto, este estudo apresenta como objetivo analisar os fatores associados ao suicídio em Hospital Psiquiátrico de Teresina, com a finalidade ulterior de elaboração de fluxos para atendimento ao paciente em situação de risco.

\section{MÉTODOS}

Trata-se de um estudo transversal analítico, realizado em um hospital psiquiátrico de Teresina, referência no tratamento de transtornos mentais graves, dentre eles pessoas com ideação e tentativa de suicídio.

A população compreendeu os pacientes atendidos no serviço de emergência ou na internação após tentativa de suicídio. Foram considerados como critérios de inclusão: ter idade igual ou superior a 18 anos e dar entrada no serviço de urgência após tentativa de suicídio. Foram excluídos os que apresentavam incapacidades clínicas que pudessem comprometer a compreensão e preenchimento dos questionários e com complicações graves que necessitavam de transferência imediata para outras instituições de saúde.

Para cálculo da amostra, foi utilizada a técnica para populações infinitas, considerando a prevalência presumida do suicídio de 10,4\%, margem de erro de $5 \%$ e nível de confiança de $95 \%$ (GIL AC, 2008). Sendo assim, a amostra mínima necessária para o estudo foi de 142 participantes e o processo de amostragem foi do tipo casual simples.

Foi considerado como variável dependente a tentativa de suicídio. As independentes foram aquelas relacionadas à caracterização sociodemográfica, clínica e terapêutica dos participantes, conforme descrito no Quadro 1. Ressalta-se que algumas variáveis foram recategorizadas para viabilizar a análise estatística.

Para coleta de dados foi utilizado um instrumento próprio, construído após revisão de literatura e seleção das melhores evidências, sendo composto por variáveis sociodemográficas (sexo, idade, escolaridade, estado civil, renda familiar, situação laboral e procedência), clínicas (local da tentativa, fatores de risco, turno, internações psiquiátricas, tipo de tentativa e sintomas presentes) e terapêuticas (encaminhamento e condutas terapêuticas).

Apesar de ser instrumento autoaplicável, tendo em vista o baixo nível da escolaridade comum na população estudada, optou-se por entrevistas que foram realizadas pelos pesquisadores com duração média de 20 minutos para cada participante em ambiente reservado e respeitando a privacidade, anonimato e sigilos das informações.

Após aprovação institucional, foi realizado contato com o serviço de admissão para levantamento dos pacientes que atendem aos critérios de inclusão, certificação do diagnóstico, do tratamento e seleção da amostra.

Os dados foram coletados mediante contato direto com os participantes do estudo, análise de prontuários médicos e fichas de atendimento. Destaca-se o desenvolvimento de treinamentos prévios com todos os pesquisadores envolvidos, a fim de dialogar sobre o instrumento que seria utilizado e padronizar a forma de abordagem aos pacientes durante o procedimento de coleta. 
Para construção da base de dados foi utilizado o software da Microsoft Office Excel e após codificação de todas as variáveis em um dicionário, empregado a técnica de validação por meio da digitação em planilha com dupla entrada. Ao serem detectadas inconsistências, os instrumentos foram localizados e as correções devidamente realizadas. Posteriormente, as informações foram transportadas para o programa STATA ${ }^{\circledR}$, visando a análise quantitativa com base nos princípios da estatística descritiva e inferencial.

Para as variáveis sociodemográficas, clínicas e terapêuticas a análise estatística foi construída por medidas de posição (média, máximo e mínimo) e de variabilidade (desvio padrão, máximo e mínimo) para as variáveis quantitativas e pela frequência absoluta (nº) e relativa (\%) para as variáveis categóricas.

$\mathrm{Na}$ análise, inferencial foi realizado o teste de Kolmogorov-Sminov para verificação da normalidade dos dados. Para verificação de associações entre as variáveis sociodemográficas, clínicas e terapêuticas com os tipos de tentativas de suicídio, foi realizado o teste exato de Fischer. Destaca-se que todas as análises foram realizadas ao nível de significância de $5 \%(p<0,05)$.

No cumprimento dos preceitos éticos e legais contidos na Resolução no 510 de 2016, do Conselho Nacional de Saúde, o projeto de pesquisa foi encaminhado para autorização institucional, em seguida, ao Comitê de Ética em Pesquisa da Universidade Federal do Piauí, via Plataforma Brasil, obtendo autorização sob parecer número 2.379.793.

Inicialmente, foram apresentados aos participantes os objetivos, métodos, riscos e benefícios do estudo por meio do Termo de Consentimento Livre e Esclarecido com linguagem clara e acessível e, ao formular o convite para participação, foi oferecido o documento em duas vias, garantindo-Ihes anonimato, privacidade e possibilidade de desistência em qualquer momento, sem penalidades ou prejuízo à assistência. Para busca de informações em prontuários e registros médicos foi solicitada autorização institucional por meio do Termo de Compromisso e Utilização dos Dados.

\section{RESULTADOS}

O estudo descritivo da amostra estudada mostrou o predomínio do sexo masculino 75 (52,8\%), com idade média de $34,4 \pm 11,0$ anos, solteiros 75 (52,8\%), desempregados $91(63,7 \%)$, com ensino fundamental $57(40,2 \%)$, católicos $76(53,5)$, renda familiar variando de um a dois salários mínimos $67(47,2 \%)$ e procedentes de Teresina 84 (59,1\%) conforme descrito na Tabela 1. 


\section{Revista Eletrônica Acervo Saúde / Electronic Journal Collection Health ｜ ISSN 2178-2091}

Tabela 1 - Caracterização sociodemográfica dos pacientes atendidos por tentativa de suicídio (n=142). Teresina-PI, 2019.

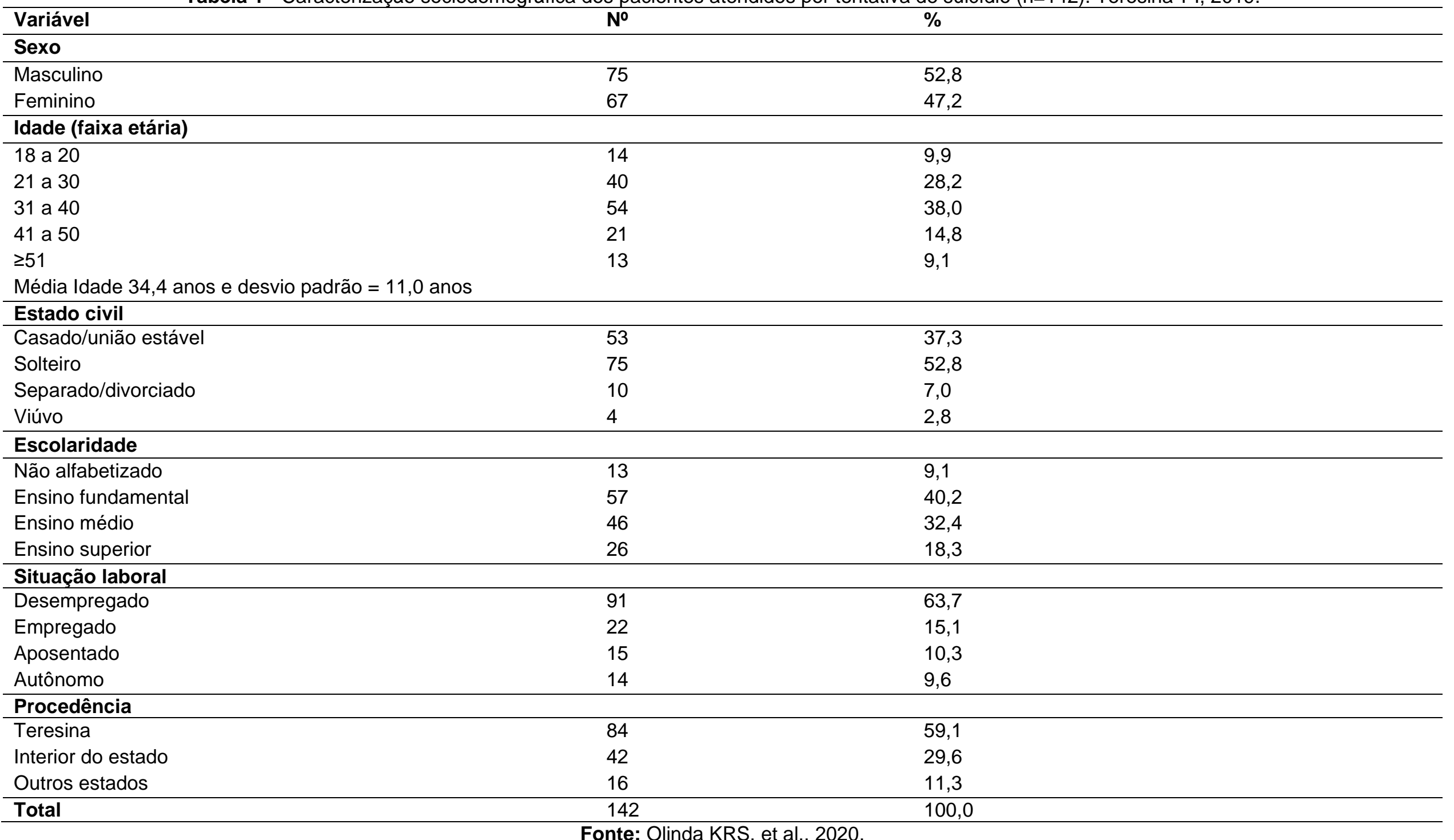

REAS/EJCH | Vol.12(6) | e3050 | DOI: https://doi.org/10.25248/reas.e3050.2020 Página $\mathbf{5}$ de $\mathbf{1 1}$ 
A Tabela 2 mostra que o ambiente domiciliar $122(85,9 \%)$ prevaleceu como o local de maior prevalência para a tentativa do suicídio, seguido de avenidas $13(9,2 \%)$ e bares $3(2,1 \%)$, especificamente nos turnos manhã $61(43,0 \%)$, tarde $61(43,0 \%)$ e noite $20(14,0 \%)$. Quanto a avaliação das condições psíquicas, verificou-se que os participantes em sua totalidade apresentaram fatores associados ao comportamento suicida, predominando o transtorno psiquiátrico observado em $80(56,3 \%)$ pessoas, seguido do consumo de substâncias psicoativas $71(50,0 \%)$, de tentativas prévias $70(49,3 \%)$, crises situacionais $62(43,7 \%)$, conflitos familiares $35(24,6 \%)$ e histórico de abuso sexual ou físico 4 (2,7\%).

Tabela 2 - Descrição do local, turno e fatores de risco para tentativa de suicídio ( $n=142)$. Teresina-PI, 2019.

\begin{tabular}{lll}
\hline Variável & № & $\%$ \\
\hline Local da tentativa & 122 & 85,9 \\
\hline Residência & 13 & 9,2 \\
Avenida & 3 & 2,1 \\
Bar & 4 & 2,8 \\
Outros & & \\
\hline Turno & 61 & 43,0 \\
\hline Manhã & 61 & 43,0 \\
Tarde & 20 & 14,0 \\
Noite & & \\
\hline Fatores de riscos & 71 & 50,0 \\
\hline Uso de SPA & 80 & 56,3 \\
Transtorno psiquiátrico & 35 & 24,6 \\
Conflitos familiars & 62 & 43,7 \\
Crises situacionais & 70 & 49,3 \\
Tentativas anteriores & 4 & 2,7 \\
Abuso sexual ou físico & 142 & 100,0 \\
\hline Total &
\end{tabular}

Legenda: № - frequência absoluta; \% - percentagem. SPA - Substância Psicoativa.

Fonte: Olinda KRS, et al., 2020.

Dentre os sintomas psíquicos presentes, verificou-se que as alterações de sensopercepção como as alucinações auditivas 99 (69,7\%) foram as mais relatadas, seguido dos delírios persecutórios $62(43,7 \%)$ e dos sintomas depressivos 54 (38,0\%). Em relação aos procedimentos terapêuticos, a internação psiquiátrica foi adotada para a maioria dos participantes 126 (88,7\%), seguido da observação no serviço de urgência 11 $(7,8 \%)$ e do encaminhamento para tratamento especializado em outros dispositivos que compões as Redes de Atenção Psicossocial 5 (3,5\%). Quanto ao tipo de tentativa, observou-se que diferentes métodos são utilizados como a intoxicação exógena por automedicação $56(39,4 \%)$ que representou o meio prevalente para o suicídio, seguido do enforcamento $45(31,7 \%)$ e da violência e lesões autoprovocadas com uso de objetos perfuro-cortantes $40(28,2 \%)$, conforme descrito na Tabela 3.

Tabela 3 - Descrição dos tipos de tentativas de suicídio ( $\mathrm{n}=142)$. Teresina-PI, 2019.

\begin{tabular}{lll}
\hline Tipos de tentativas & № & $\%$ \\
\hline Automedicação & 56 & 39,4 \\
Enforcamento & 45 & 31,7 \\
Arma de fogo & 7 & 4,9 \\
Altura & 11 & 7,8 \\
Objetos perfuro-cortantes & 40 & 28,2 \\
Outros & 12 & 8,4 \\
\hline Total & 142 & $100,0^{*}$ \\
\hline
\end{tabular}

Legenda: № - frequência absoluta; \% - percentagem. Frequências apresentadas para categoria sim. *A soma das frequências são maiores que $100 \%$, pois o mesmo paciente pode ter usado mais de um meio para a tentativa de suicídio. Fonte: Olinda KRS, et al., 2020. 
A análise bivariada entre as variáveis categóricas independentes (sociodemograficas, clínicas e terapêuticas) e a variável dependente (tipo de tentativa de suicídio), mostrou que o sexo ( $p=0,001)$, a idade dos participantes $(p<0,005)$, o local da tentativa $(p=0,001)$, o histórico de tentativas prévias $(p=0,003)$ e a internação psiquiátrica $(p=0,009)$ apresentaram associações estatisticamente significativas com 0 tipo de tentativa de suicídio. As associações entre as variáveis estão contempladas nas Tabela 4.

Tabela 4 - Associação do tipo de tentativa de suicídio com as variáveis sóciodemográficas. Teresina-PI, 2019.

\begin{tabular}{|c|c|c|c|c|c|c|}
\hline $\begin{array}{l}\text { Variável/Tipo de } \\
\text { tentativa }\end{array}$ & $\begin{array}{l}1 \\
\text { № (\%) }\end{array}$ & $\begin{array}{l}\mathbf{2} \\
\text { № (\%) }\end{array}$ & $\begin{array}{l}3 \\
\text { № (\%) }\end{array}$ & $\begin{array}{l}4 \\
\text { № (\%) }\end{array}$ & $\begin{array}{l}5 \\
\text { № (\%) }\end{array}$ & p-valor \\
\hline Sexo \# & & & & & & 0,001 \\
\hline Masculino & $11(35,5)$ & $23(82,1)$ & $17(68,0)$ & $13(43,3)$ & $10(38,5)$ & \\
\hline Feminino & $20(64,5)$ & $05(17,9)$ & $08(32,0)$ & $17(53,7)$ & $16(61,5)$ & \\
\hline Idade*\# (faixa etária) & & & & & & $<0,005$ \\
\hline 18 a 20 & $1(3,2)$ & $2(7,1)$ & $2(8,0)$ & $5(16,7)$ & $3(11,5)$ & \\
\hline 21 a 30 & $12(38,7)$ & $3(10,7)$ & $9(36,0)$ & $7(23,3)$ & $9(34,6)$ & \\
\hline 31 a 40 & $8(25,8)$ & $20(71,5)$ & $9(36,0)$ & $7(23,3)$ & $10(38,5)$ & \\
\hline 41 a 50 & $8(25,8)$ & $2(7,1)$ & $3(12,0)$ & $5(16,7)$ & $3(11,5)$ & \\
\hline$\geq 51$ & $2(6,4)$ & $1(3,6)$ & $2(8,0)$ & $6(20,0)$ & $1(3,9)$ & \\
\hline Estado civil ${ }^{*}$ & & & & & & 0,750 \\
\hline União estável & $2(6,4)$ & $1(3,6)$ & $2(8,0)$ & $6(20,0)$ & $1(3,9)$ & \\
\hline Solteiro & $9(29,0)$ & $11(39,3)$ & $10(40,0)$ & $11(36,7)$ & $11(42,3)$ & \\
\hline Separado & $18(58,1)$ & $15(53,5)$ & $15(60,0)$ & $16(53,3)$ & $10(38,5)$ & \\
\hline Viúvo & $3(9,7)$ & $1(3,6)$ & - & $2(6,7)$ & $4(15,4)$ & \\
\hline Escolaridade $^{\star}$ & & & & & & 0,962 \\
\hline Não alfabetizado & $5(16,1)$ & $1(3,6)$ & $2(8,0)$ & $3(10,0)$ & $1(3,8)$ & \\
\hline Ensino fundamental & $10(32,3)$ & $12(42,9)$ & $11(44,0)$ & $12(40,0)$ & $12(46,2)$ & \\
\hline Ensino médio & $11(35,5)$ & $10(35,7)$ & $8(32,0)$ & $9(30,0)$ & $7(26,9)$ & \\
\hline Ensino superior & $5(16,1)$ & $5(17,9)$ & $4(16,0)$ & $6(20,0)$ & $6(23,1)$ & \\
\hline Religião* & & & & & & 0,744 \\
\hline Católico & $16(51,6)$ & $14(50,0)$ & $14(56,0)$ & $14(46,7)$ & $16(61,5)$ & \\
\hline Evangélico & $9(29,1)$ & $11(39,3)$ & $9(36,0)$ & $8(26,7)$ & $8(30,7)$ & \\
\hline Espírita & $1(3,2)$ & - & - & $1(3,3)$ & $1(3,9)$ & \\
\hline Outro & $5(16,1)$ & $3(10,7)$ & $2(8,0)$ & $7(23,3)$ & $1(3,9)$ & \\
\hline Situação laboral ${ }^{*}$ & & & & & & 0,718 \\
\hline Desempregado & $22(71,0)$ & $19(67,9)$ & $17(68,0)$ & $16(53,3)$ & $16(61,6)$ & \\
\hline Empregado & $2(6,4)$ & $4(14,3)$ & $5(20,0)$ & $5(16,7)$ & $5(19,2)$ & \\
\hline Aposentado & $4(12,9)$ & $1(3,5)$ & $2(8,0)$ & $6(20,0)$ & $2(7,7)$ & \\
\hline Autônomo & $3(9,7)$ & $4(14,3)$ & $1(4,0)$ & $3(10,0)$ & $3(11,5)$ & \\
\hline Renda familiar* & & & & & & 0,572 \\
\hline Sem renda & $5(16,1)$ & $5(17,9)$ & $3(12,0)$ & $4(13,3)$ & $1(3,9)$ & \\
\hline$<1$ & $7(22,6)$ & $4(14,3)$ & $4(16,0)$ & $5(16,7)$ & $9(34,6)$ & \\
\hline 1 a 2 & $16(51,6)$ & $11(39,2)$ & $14(56,0)$ & $13(43,3)$ & $11(42,3)$ & \\
\hline 3 a 5 & $3(9,7)$ & $8(28,6)$ & $4(16,0)$ & $8(26,7)$ & $5(19,2)$ & \\
\hline Procedência* & & & & & & 0,684 \\
\hline Teresina & $16(51,6)$ & $16(57,2)$ & $19(76,0)$ & $16(53,3)$ & $15(57,7)$ & \\
\hline Interior do estado & $10(32,3)$ & $10(35,7)$ & $5(20,0)$ & $9(30,0)$ & $8(30,8)$ & \\
\hline Outros estados & $5(16,1)$ & $2(7,1)$ & $1(4,0)$ & $5(16,7)$ & $3(11,5)$ & \\
\hline Total & $31(100,0)$ & $28(100,0)$ & $25(100,0)$ & $30(100,0)$ & $26(100,0)^{*}$ & \\
\hline
\end{tabular}

Legenda: * Teste exato de Fisher; \# valor de $p<0,05$; 1 - Automedicação; 2 - Enforcamento; 3 - Arma de fogo/Altura/ Outras substâncias/outras; 4 - Objeto perfuro cortante; 5 - Mais de 1 tipo de tentativas diferentes.

Fonte: Olinda KRS, et al., 2020. 


\section{DISCUSSÃO}

Neste estudo a prevalência da tentativa de suicídio foi no sexo masculino, diferindo de outras pesquisas nacionais e internacionais que apontaram maior ocorrência em mulheres (SHARMA B, et al., 2015; CLAUMANN GS, et al., 2018). Esse resultado pode ser justificado pela internação psiquiátrica prolongada, conduta adotada para maioria dos pacientes e descrita como fator preditor para o agravo, além do perfil institucional que atende maior demanda do sexo masculino (FRANKLIN JC, et al., 2017).

A prevalência de tentativa de suicídio nos homens associa-se ainda a maior predisposição para desenvolvimento de transtornos mentais e comportamentais, sejam eles esquizofrênicos, de humor ou relacionados ao uso de álcool e outras drogas, bem como aos desajustes causados por relacionamentos familiares conturbados, violência intrafamiliar, doenças físicas incapacitantes e condições desfavoráveis de trabalho (SENA FN, et al., 2014).

Em relação à idade, verificou-se que a maior parte da amostra foi composta por adultos jovens, com idade média de $34,4(11,0)$ anos. O predomínio dessa faixa etária reitera a idade como um dos principais fatores predisponentes, tendo em vista a dificuldade para lidar com fatores estressores (SESAPI, 2018).

Destaca-se que o quantitativo de adolescentes foi expressivo corroborando com os levantamentos realizados pela OMS e refletindo as modificações psicológicas, físicas e sociais associadas à puberdade que são caracterizadas por movimentos de dependência e independência extrema, períodos de contradições, conflitos e ambivalências. Nesse sentido, em busca da formação de identidade pessoal, tornam-se comuns a presença de sintomas de baixa autoestima, ansiedade e depressão, levando a comportamentos atípicos como a tentativa de suicídio que põem em risco a vida do indivíduo (DUCHESNE AP, et al., 2017; SIABATO MEF, et al., 2017).

Estudo que avaliou os dados epidemiológicos do suicídio na população jovem, comprovou a elevação dos indicadores de incidência em 15,3\%, passando de 2.515 casos para 2.900 no período de 2002 à 2012 . Sendo assim, a taxa de suicídio, para essa população, passou de 5,1 para 5,6/100 mil jovens, levando o país a ocupar a 60ª posição na classificação mundial (WAISELFISZ JJ, 2014).

Quanto à situação conjugal, houve o predomínio de solteiros que pode estar relacionado tanto à idade dos participantes, quanto às dificuldades em manter uma rede de relacionamentos, tendo em vista que a maioria apresentou transtorno mental prévio. Essa condição representa fator de risco para a tentativa de suicídio, uma vez que o cônjuge representa referenciais para reestruturação de vínculos afetivos, bem como para manutenção da segurança no manejo da doença, do tratamento e de incapacidades, constituindo assim, fonte de vigilância, apoio social e emocional (VIDAL CAL, GONTIJO ECM, LIMA AL, 2013).

A baixa escolaridade reflete a realidade brasileira no segmento educacional, em que as dificuldades nas informações, as deficiências para reconhecimento dos fatores de risco e as limitações no acesso aos serviços de saúde levam ao aumento dos indicadores de incidência e mortalidade, bem como ao comprometimento do autocuidado devido à presença de incapacidades temporárias ou permanentes e a necessidade de intervenções agressivas (VIDAL CAL, GONTIJO ECM, LIMA AL, 2013).

Com relação à situação laboral, houve o predomínio de desempregados, com a renda familiar entre 1 e 2 salários mínimos, o que pode revelar a influência do padrão de dependência nas relações sociais, bem como a baixa escolaridade predominante nessa população. Destaca-se que na amostra estudada a maioria dos participantes eram pessoas em idade produtiva e que o desemprego também pode refletir a necessidade de redefinição dos padrões de vida, o contexto neoliberal contemporâneo que exige a multifuncionalidade dos trabalhadores, e os estigmas frente a temática, sendo constantemente associada à inutilidade laboral (CANTAO L, BOTTI NCL, 2016).

Assim, as dificuldades financeiras evidenciam as desigualdades sociais, econômicas e culturais do Brasil, podendo levar ao comprometimento psicossocial e limitar o acesso aos serviços de saúde, bem como acarretar 0 atraso diagnóstico de comorbidades psiquiátricas, impossibilitando a aquisição de terapias adjuvantes e gerando impactos na economia familiar, instabilidades econômicas e perda da produtividade (FELIX TA, et al., 2016). 
Em virtude da ausência de infraestrutura, recursos adequados e de centros de referências para tratamento de transtornos mentais graves, muitos pacientes procedentes do interior do Piauí e de outros estados realizam acompanhamento em Teresina, gerando mudanças na rotina de vida, distanciamento familiar, aumento dos custos com a doença e interferindo na rede de apoio e suporte social.

Quanto à caracterização clínica, verificou-se que o comportamento suicida é um problema complexo e multifacetado, tendo a elevação das taxas de incidência constantemente associada à presença de transtornos mentais crônicos e graves, incluindo as alterações comportamentais decorrentes do uso abusivo de álcool e de outras substâncias psicoativas que potencializam o risco para ocorrência do evento em 6,5 a 9 vezes (ARRIBAS-IBAR E, et al., 2017).

A prevalência de transtornos mentais verificada em $56,3 \%$ dos casos aumenta o estado de vulnerabilidade para o suicídio, sendo também evidenciada em outros estudos que apontaram associações significativas entre as comorbidades psiquiátricas e o alto risco, dentre elas a depressão, a esquizofrenia e as alterações de humor, de personalidade e de comportamento (ARRIBAS-IBAR E, et al., 2017).

A literatura demonstra que os transtornos de humor predominam quando associadas ao comportamento suicida, seguido do uso de substâncias psicoativas e que a situação de risco é agravada quando diferentes condições se combinam, como, por exemplo, a depressão e o alcoolismo, ou quando há coexistência de diferentes sintomas psiquiátricos como ansiedade e agitação psicomotora (PIRES MCC et al., 2015).

O consumo de substâncias psicoativas verificado na amostra estudada transita fortemente por diferentes grupos sociais, faixas etárias e gêneros, trazendo impactos sociais, econômicos, penais e na saúde física e psicológica dos indivíduos, assim como na ampliação de indicadores de morbimortalidade constantemente associados às condições de violência autoprovocada (VARGAS D, et al., 2015).

A exposição às drogas, sejam elas lícitas ou ilícitas, se configura como fator de risco e situação de vulnerabilidade ao comportamento suicida, estando em diferentes estudos diretamente associado à ideação, ao planejamento ou à tentativa de suicídio. Embora considerado um fenômeno historicamente relacionado aos homens, nos últimos anos, tem-se observado que o consumo de SPA passou a ser prática frequentemente entre as mulheres, principalmente no período da adolescência, constantemente permeado por dúvidas, instabilidades emocionais e crises de identidade (MALTA DC et al., 2014).

Atualmente, a dependência e o abuso dessas substâncias se caracterizam como um problema de saúde pública de abrangência mundial, uma vez que favorece o desenvolvimento de comorbidades psicopatológicas que podem contribuir para perda de valor existencial e consequentemente determinar o comportamento suicida e a situação de risco (FELIX TA, et al., 2016, VARGAS D, et al., 2015).

Outra condição associada ao comportamento suicida, foi a presença de tentativas prévias evidenciada em $49,3 \%$ dos participantes. A literatura aponta que esse aspecto é comum nos pacientes, devendo ser clinicamente investigado, tendo em vista que uma tentativa prévia constitui um dos principais fatores de risco para a futura efetivação desse intento. Nesse sentido, as tentativas devem ser reconhecidas como emergências psiquiátricas e sinal de alerta para indicar a possibilidade de fenômenos psicossociais complexos (BOTEGA JN, 2014).

Os conflitos familiares $(24,6 \%)$ e crises situacionais como conflitos conjugais foram determinantes para a tentativa de suicídio, uma vez que sentimentos de vulnerabilidade, desamparo e frustração prejudicaram as relações familiares e contribuíram para estados de conflitos e desarmonia (MEDEIROS KT, et al., 2013). Nessa perspectiva, a negligência e o abandono no ambiente familiar refletem a necessidade de apoio social como importante estratégia de suporte para prevenção do agravo e identificação de comportamentos e atitudes de risco (MILLER P, et al., 2019; CANTAO L, BOTTI NCL, 2016).

Em relação aos meios utilizados, podem variar segundo aspectos socioculturais, facilidades no acesso, gênero, faixa etária e presença de tentativas recorrentes. No estudo foi observado que a intoxicação exógena prevaleceu, corroborando com os resultados encontrados por Silva ES, Marques JJ e Suchara EA (2018). O enforcamento e a violência autoprovocada com objetos perfuro-cortantes também foram descritos como causas frequentes para o suicídio, assim como em outros estudos (CUNHA FA, et al., 2016). 
Ressalta-se que a intoxicação e o enforcamento representam os meios de escolha pelo fácil acesso e disponibilidade, e que na maioria das vezes as estratégias para prevenção são efetivadas em ambientes institucionais por meio de condutas medicamentos e medidas de vigilância (BOTEGA JM, et al., 2012). Desse modo, os métodos terapêuticos devem ser iniciados precocemente tendo em vista que a dor de difícil controle, estados metabólicos anormais e condições que afetam o sistema nervoso central, favorecem o aumento dos indicadores de incidência e mortalidade por suicídio (BOTEGA JM, et al., 2012).

A internação psiquiátrica adotada como conduta para a maioria dos pacientes representa uma medida assistencial integral, de ação curativa e preventiva, bem como fornece indicadores que caracterizam o suicídio como problema de saúde pública. No entanto, quando prolongada, pode potencializar o risco para violência autoprovocada devido o distanciamento familiar, a exacerbação de sintomas de ansiedade e os estados de abstinência (FRANKLIN JC, et al., 2017).

A variável sexo apresentou associação $(p=0,001)$ com meio utilizado para tentativa de suicídio, sendo também relatada em outro estudo, em que nos homens ocorreu o predomínio do enforcamento e da lesão por arma de fogo e em mulheres a intoxicação exógena, seja por automedicação ou por outras substâncias (MACHADO DB, SANTOS DN, 2015). O estudo de Vidal CAL, Gontijo ECM e Lima AL (2013), mostrou que técnicas letais são observadas com maior frequência entre os homens, sendo o enforcamento o principal meio utilizado, reafirmando que os indicadores de suicídio se concentram no sexo masculino.

O histórico de tentativas prévias ( $p=0,003$ ) também apresentou associação significativa, se configurando parâmetro para determinação do risco. Estudo epidemiológico estimou que até $60 \%$ dos atendimentos por tentativa de autoextermínio apresentam tentativas prévias e que $10 \%$ a $25 \%$ poderão tentar em prazo médio de um ano (BERTOLOTE JM, et al., 2005). O comportamento suicida durante internação psiquiátrica foi expressivo, podendo ser refletir a fragilidade dos vínculos familiares, sociais e laborais, nível de ansiedade e depressão, medo de recorrência de doenças e distanciamento de grupos de apoio (BOTEGA JN, 2014).

Destaca-se que em casos de internações na modalidade compulsória, o problema torna-se ainda mais grave, pois o paciente permanece em ambiente hospitalar mesmo após alta médica ficando assim susceptível à recaídas, além de estar submetido, na maioria das vezes, às condições insalubres e à possibilidade de retorno ao sistema prisional uma vez que tenha cometido crimes.

\section{CONCLUSÃO}

Além de confirmar o suicídio como um problema de saúde pública de grande magnitude, os resultados evidenciaram que diferentes condições determinam a situação de risco, sendo prevalentes o diagnóstico prévio de transtornos psiquiátricos, o consumo de substâncias psicoativas, o sexo, idade e o histórico de tentativas anteriores e internações prévias. Mostra-se necessária a realização de novos estudos visando a estruturação de serviços especializados para direcionando do tratamento, aplicação de medidas efetivas para prevenção de recaídas e de estratégias para monitorização e controle do comportamento.

\section{REFERÊNCIAS}

1. ARRIBAS-IBAR E, et al. Suicidal behaviours in male and female users of illicit drugs recruited in drug treatment facilities. Gac Sanit., 2017: 31(4): 292-8.

2. BAHIA CA, et al. Lesão autoprovocada em todos os ciclos da vida: perfil das vítimas em serviços de urgência e emergência de capitais do Brasil. Ciência \& Saúde Coletiva, 2017; 22(9): 2841-2850.

3. BERTOLOTE JM, et al. Suicide attempts, plans, and ideation in culturally diverse sites: The WHO SUPRE-MISS community survey. Psychological Medicine, 2005; 35: 1457-65.

4. BOTEGA NJ. Prática psiquiátrica no hospital geral: Interconsulta e emergência. $3^{\circ}$ Ed..Porto Alegre: Artmed, 2011.

5. BOTEGA JN. Comportamento suicida: epidemiologia. Psicologia USP, 2014; 25(3): 231-236.

6. CALIXTO M, ZERBINI T. Epidemiologia do suicídio no Brasil entre os anos de 2000 e 2010. Saúde, Ética \& Justiça, 2016; $21(2): 45-51$.

7. CANTÃO L, BOTTI NCL. Suicidal behavior among drug addicts. Rev Bras Enferm., 2016; 69(2): 366-73.

8. CARDOSO HF, et al. Suicídio no Brasil e América Latina: revisão bibliométrica na base de dados Redalycs. Diaphora, $2014 ; 12(2): 42-48$. 
9. CLAUMANN GS, et al . Prevalência de pensamentos e comportamentos suicidas e associação com a insatisfação corporal em adolescentes. J. Bras. Psiquiatr., 2018; 67(1):3-9.

10. CUNHA FA, et al. Análise documental sobre os suicídios ocorridos na região de Jundiaí entre 2004 e 2014 . Salud Soc., 2016; 7(2): 212-22.

11. DUCHESNE AP, et al. Body dissatisfaction and psychological distress in adolescents: Is self-esteem a mediator? J Health Psychol., 2017; 22(12):1563-9.

12. FELIX TA, et al. Fatores de risco para tentativa de suicídio: produção de conhecimento no Brasil. Contexto Saúde., $2016 ; 16(31): 173-85$.

13. FRANKLIN JC, et al. Risk factors for suicidal thoughts and behaviors: A meta-analysis of 50 years of research. Psychol Bull., 2017; 2: 187-232.

14. GIL AC. Métodos e técnicas de pesquisa social. 6 ed. São Paulo: Atlas, 2008.

15. MACHADO DB, SANTOS DN. Suicídio no Brasil, de 2000 a 2012. J Bras Psiquiatr., 2015; 64(1): 45-54.

16. MALTA DC, et al. Alcohol consumption among Brazilian Adolescents according to the National Adolescent Schoolbased Health Survey (PeNSE 2012). Rev Bras Epidemiol, 2014; 17: 203-14.

17. MEDEIROS KT. et al. Social representations of the use and abuse of drugs among relatives of users. Psicol Estud., $2013 ; 18(2): 269-79$.

18. MILLER P, et al. Depression, suicide risk, and workplace bullying: a comparative study of fly-in, fly-out and residential resource workers in Australia. Aust Health Ver., 2019; 13:1-10.

19. MULLER AS, PEREIRA G, ZANON RB. Estratégias de prevenção e pósvenção do suicídio: Estudo com profissionais de um Centro de Atenção Psicossocial. Rev. Psicol. IMED, 2017; 9(2):6-23.

20. PIRES MCC, et al. Risk indicators for attempted suicide for poisoning: a study case-control. J Bras Psiquiatr., 2015; 64(3):193-9.

21. SENA FN, et al. Fatores de risco relacionados com suicidios em Palmas (TO), Brasil, 2006-2009, investigados por meio de autopsia psicossocial. Cad. Saud. Col. 2014; 19(1):115-26.

22. SESAPI. Secretaria de Estado da Saúde do Piauí. Perfil epidemiológico da mortalidade por suicídio e violência autoprovocada (tentativa de suicídio) no estado do Piauí, no período de 2015 a 2017. p. 1-15, 2018.

23. SHARMA B, et al. Factors Associated with Suicidal Ideation and Suicide Attempt among School-Going Urban Adolescents in Peru. Int J Environ Res Public Health., 2015; 12(11): 14842-56.

24. SIABATO MEF. et al.. Asociación entre depresión e ideación suicida en un grupo de adolescentes colombianos. Pensam Psicol., 2017; 15(1):51-61.

25. SILVA ES; MARQUES JJ, SUCHARA EA. Perfil de suicídios em município da Amazônia Legal. Cad. saúde colet., $2018 ; 26(1): 84-91$.

26. SILVA TP, LIMA MD, SOUGEY EB. Alucinógenos, anfetaminas e comportamento suicida: revisão integrativa da literatura. Rev Hosp Univ Pedro Ernesto., 2016; 15(1):28-36.

27. SOUZA VS, et al. Tentativas de suicídio e mortalidade por suicídio em um município no interior da Bahia. J Bras Psiquiatr., 2011; 60(4):294-300.

28. VARGAS D, et al. O primeiro contato com as drogas: análise do prontuário de mulheres atendidas em um serviço especializado. Saúde Debate, 2015; 39(106):782-91.

29. VIDAL CAL, GONTIJO ECM, LIMA AL. Tentativas de suicídio: fatores prognósticos e estimativa do excesso de mortalidade. Cad. Saúde Pública, Rio de Janeiro, 2013; 29(1):175-187.

30. WAISELFISZ JJ. Mapa da violência 2014: os jovens do Brasil. Brasília, 2014.

31. WORLD HEALTH ORGANIZATION. Preventing suicide: a global imperative [internet]. Geneve: World Health Organization, 2014, 92p.

32. WORLD HEALTH ORGANIZATION. Preventing suicide: a community engagement toolkit. Pilotversion. Geneva: World Health Organization, 2016.

33. YARBOROUGH BJH, et al. Challenges of Population-based Measurement of Suicide Prevention Activities Across Multiple Health Systems. EGEMS (Wash DC)., 2019; 7(1):1-123. 